\title{
INEQUALITIES ON POLYNOMIAL ROOTS
}

\author{
DORU ŞTEFĂNESCU
}

Abstract. The paper presents a survey of inequalities involving roots of univariate polynomials with complex coefficients. These allow improvements in the methods of Bernoulli and Graeffe. Inequalities involving the length of a polynomial are also deduced.

Mathematics subject classification (2000): 12D10, 26C05, 30C10.

Key words and phrases: Inequalities on polynomials, roots, length.

\section{REFERENCES}

[1] D. Bernoulli: Observationes de Seriebus, Comment. Acad. Sci. Petrop., 3, 85-100, 1728 (1732).

[2] W. S. CASSEls: An Introduction to Diophantine Approximation, Cambridge Univ. Press, (1957).

[3] A.-L. CAUCHY: Exercices de Mathématiques, t. 4, Paris (1829).

[4] L. Cerlienco, M. Mignotte, F. Piras: Suites linéaires récurrentes, Enseign. Math., 33, 69-81 (1987).

[5] J. H. DaVenport, M. Mignotte: On finding the largest root of a polynomial, Math. Modelling Num. Anal., 24, 693-696 (1990).

[6] C. F. GRAEFFE: Beweis eines Satzes aus der Theorie der numerischen Gleichungen, J. reine angew. Math., 10, 288-291 (1833) 288-291.

[7] P. HENRICI: Elements of Numerical Analysis, Wiley, New York (1964).

[8] P. HenRICI: Computational Complex Analysis, vol. 2, Addison-Wesley, Reading Ma. (1976).

[9] A. S. Householder: Dandelin, Lobachevskii or Graeffe?, Amer. Math. Monthly, 66, 464-466 (1959).

[10] G. JACOBI: Observatiunculae ad theoriam æquationum pertinenses (1834), in Gesammelte Werke, Bd. 3, 271-284, Reimer Verlag, Berlin (1884).

[11] D. KNUTH: The Art of Computer Programming, Volume 2: Seminumerical Algorithms, AddisonWesley, Reading Ma (1981).

[12] M. MARdEn: Geometry of Polynomials, AMS Math. Surv. and Monographs, Providence (1989).

[13] M. MignotTE: An inequality on the greatest roots of a polynomial, Elem. d. Math., 3, 86-87 (1991).

[14] M. Mignotte, D. ŞTEFĂNescu: Polynomials, an algorithmic approach, Springer Verlag, SingaporeBerlin (1999).

[15] M. Mignotte, D. ŞTEFĂNESCU: Estimates for polynomial roots, Appl. Algebra Eng. Comm. Comput. 12, 437-453 (2001).

[16] M. MignotTe, D. ŞTEFĂNESCU: Linear recurrent sequences and polynomial roots, IRMA, Strasbourg, 21/2001, 1-18 (2001).

[17] I. NewTON: Arithmetica universalis, Cambridge (1707).

[18] W. SPECHT: Zur Theorie der algebraischen Gleichungen, Jahr. DMV, 48, 142-145 (1938).

[19] H. WEBER: Lehrbuch der Algebra, bd. I, Braunschweig (1895). 